\title{
Prevalence of chlamydial eye infection in patients attending an eye clinic, a VD clinic, and in healthy
} persons

\author{
ROLF RÖNNERSTAM,' KENNETH PERSSON, ${ }^{2}$ HOLGER HANSSON, ${ }^{3}$ AND \\ KAY RENMARKER ${ }^{4}$
}

From the departments of 'Ophthalmology, ${ }^{2}$ Clinical Virology, ${ }^{3}$ Dermato-Venereology, and ${ }^{4}$ Personnel Health Care, University of Lund, Malmö General Hospital, Malmö, Sweden

SUMmaRY Chlamydial eye infection was detected in 28 of 983 ophthalmological patients with conjunctivitis or keratoconjunctivitis, with a peak frequency of over $9 \%$ in patients aged 16-20 years and with decreasing frequency thereafter. In patients aged 1 to 15 years chlamydial conjunctivitis was not observed. Chlamydial eye infection could not be detected in patients at a venereal diseases clinic, though chlamydial genital infection was rather frequent in these patients. Nor was Chlamydia trachomatis found in the eyes of healthy young adults. In patients with proved chlamydial conjunctivitis unilateral symptoms were the rule. Pseudoptosis was the most conspicuous presentation in two cases. A prolonged course can be expected in chlamydial eye infection if the condition is unrecognised and effective treatment delayed. The venereal background of the condition must also influence the management.

Infections by Chlamydia trachomatis are now considered to be one of the major causes of sexually transmitted diseases in the industrialised countries. Besides genital infections, which constitute a considerable public health problem, $C$. trachomatis may cause eye infections. Chlamydial eye infections have been observed in sexually active young adults and in neonates, where the infection has been transmitted to the infant from the mother. The clinical features include conjunctivitis with papillary and follicular reaction (inclusion conjunctivitis); corneal lesions (keratitis punctata); and invasion of the cornea by blood vessels (pannus), a condition indistinguishable from trachoma. ${ }^{2}$ Tarsal scarring may eventually develop in some cases. Follicles are seldom found in infants, as tarsal lymphoid tissue is scarce. Chlamydial disease of sexual origin with genital and eye manifestations has been designated paratrachoma. Classical endemic trachoma can usually be separated from paratrachoma on epidemiological, serological, and often clinical grounds. Endemic trachoma is usually seen as an intrafamilial infection

Correspondence to Rolf Rönnerstam, MD, Department of Ophthalmology, Nässjö Hospital, S-571 00 Nässjö, Sweden. in underprivileged areas of the world, with the highest incidence in early childhood. ${ }^{3}$ Recurrences and reinfections, usually within the family group, are part of the disease. Chlamydial strains isolated from patients with endemic trachoma usually belong to the serovarieties $\mathrm{A}, \mathrm{B}, \mathrm{Ba}$, and $\mathrm{C}$. Strains of genital origin can generally be assigned to the serovarieties D-K, with few exceptions. ${ }^{4}$

The causative role of $C$. trachomatis in inclusion conjunctivitis has been established by experimental inoculation of the eyes of volunteers. ${ }^{5}$ The venereal nature of inclusion conjunctivitis has also been repeatedly confirmed. ${ }^{\circ \rightarrow}$ In some situations, however, eye-to-eye transmission seems likely or at least has been suggested - for example, siblings and relatives to infants with inclusion blenorrhea, and medical personnel. - $^{-11}$ Chlamydial eye infection has also been associated with swimming pool bathing. ${ }^{12}$

In this investigation clinical and epidemiological aspects of chlamydial eye infections were studied in patients attending an eye clinic because of symptoms of conjunctivitis. The prevalence of $C$. trachomatis eye infection in patients at a venereal diseases clinic and in a group of healthy young adults was also assessed. 


\section{Materials and methods}

Ophthalmological patients. Patients seen at the Department of Ophthalmology at Malmö General Hospital with conjunctivitis and keratitis were included in the study. For practical reasons all patients with conjunctivitis could not be examined for chlamydial infection. Although there was no conscious selection of patients, a bias was probably introduced in the material because patients with longstanding symptoms, and therefore with repeated visits to the department, would be more likely to have a chlamydial culture than the average patient. The department serves the whole city of Malmö, with a population of a quarter of a million people. For patients with acute eye symptoms few other medical facilities are available, and therefore patients seen at the hospital are likely to constitute a representative cross-section of the community.

Eye swabs for chlamydial isolation were obtained from 983 patients during a period of four years, and all these patients were examined with a corneal microscope.

$S T D$ patients. All patients attending the clinic for sexually transmitted diseases (STD patients) with suspected venereal disease were examined for chlamydial eye infection in addition to genital culture. During four months 855 patients were studied. A cotton-wool swab was obtained from each eye.

Healthy young adults. Healthy persons aged 16 to 25 years seeking public employment were examined by eye swabs for chlamydial eye infection at a routine medical check-up. Altogether 127 persons were examined.

Chlamydial isolation. Cotton-wool tipped swabs were used to get specimens for isolation of $C$. trachomatis. Swabs were placed in a transport medium of sucrose-containing phosphate buffer ( 2
SP), which was used to inoculate cell cultures of irradiated McCoy cells on round cover slips. Each specimen was inoculated in duplicate. After incubation at $37^{\circ} \mathrm{C}$ for 72 hours typical chlamydial inclusions were detected after iodine staining.

\section{Results}

Ophthalmological patients. Of the 983 patients examined for C. trachomatis in the eyes $61 \%$ were females; they included patients of all ages from 1 year upwards. Chlamydial eye infection was demonstrated in 28 patients, of whom $64 \%$ were females. As seen in Table 1 chlamydial eye infection was not detected in patients under 16 years of age or older than 40 years. The highest frequency of chlamydial eye infection of over $9 \%$ was found at ages 16-20 years. The frequency decreased gradually with increasing age. The mean age of the patients with chlamydial eye infection was 23 years. Microbiological examination in 26 of the chlamydia positive cases did not show any additional bacterial pathogen in the eyes. A genital examination was performed on nine of the 18 females with chlamydial conjunctivitis, and genital chlamydial infection was detected in five of these patients. A single male of the 10 with positive eye culture was also examined by urethral culture which yielded $C$. trachomatis.

Clinical symptoms from the eyes in chlamydial infection were unilateral in 20 cases, and in the remaining eight cases with bilateral infection symptoms were generally more pronounced in one eye. Follicular conjunctivitis was regularly seen in cases of some duration. Corneal lesions observed included keratitis punctata, and marginal as well as central infiltrates clinically similar to those associated with adenovirus infection. Vascular infiltration of the cornea was not observed. Two cases had unilateral pseudoptosis as the most conspicuous symptom.

Table 1 Chlamydial eye and genital infection according to age in different patient categories

\begin{tabular}{|c|c|c|c|c|}
\hline \multirow[t]{2}{*}{$\begin{array}{l}\text { Age groups } \\
\text { (years) }\end{array}$} & \multirow[t]{2}{*}{$\begin{array}{l}\text { Ophthalmologic patients: } \\
\text { chlamydial conjunctivitis, } \\
\text { number examined }\end{array}$} & \multicolumn{2}{|c|}{$\begin{array}{l}\text { VD patients: } \\
\text { genital chlamydial infection, } \\
\text { number examined }\end{array}$} & \multirow[t]{2}{*}{$\begin{array}{l}\text { Healthy adults: } \\
\text { chlamydial eye infection, } \\
\text { number examined }\end{array}$} \\
\hline & & females & males & \\
\hline $1-5$ & $0 / 90$ & - & - & - \\
\hline $6-10$ & $0 / 40$ & - & - & - \\
\hline $16-20$ & $13 / 147(9 \cdot 3 \%)$ & $23 / 84(27 \%)$ & $18 / 78(23 \%)$ & $0 / 77$ \\
\hline $21-25$ & $8 / 153(5 \cdot 2 \%)$ & $19 / 76(25 \%)$ & $34 / 155(22 \%)$ & $0 / 50$ \\
\hline $26-30$ & $5 / 123(4 \cdot 1 \%)$ & $6 / 53(11 \%)$ & $20 / 131(15 \%)$ & - \\
\hline $31-35$ & $1 / 84(1 \cdot 2 \%)$ & $1 / 21(5 \%)$ & $19 / 97(20 \%)$ & - \\
\hline $36-40$ & $1 / 67(1 \cdot 5 \%)$ & $2 / 19(11 \%)$ & $10 / 46(22 \%)$ & - \\
\hline $41-$ & $0 / 240$ & $1 / 21(5 \%)$ & $3 / 65(5 \%)$ & - \\
\hline Total & $28 / 983(2 \cdot 8 \%)$ & $55 / 281(19 \cdot 6 \%)$ & $104 / 574(18 \cdot 1 \%)$ & $0 / 127$ \\
\hline
\end{tabular}


Eye symptoms had persisted for days to several months when chlamydial eye infection was confirmed. The mean duration of symptoms was about five weeks when proper diagnosis was secured.

One of the women developed chlamydial eye infection a few days after the same condition had been diagnosed in her baby. A male patient revealed that he and his fiancé had the same symptoms from the eyes, but a chlamydial isolate could be secured only from the man, as his girl friend lived in another town.

Patients with chlamydial eye infection were treated with oral doxycyclin $100 \mathrm{mg}$ a day for nine days. One nursing mother was given erythromycin $1 \mathrm{~g}$ a day instead. Topical chloramphenicol or chlortetracycline hydrochloride eye ointment was also used regularly to prevent possible superinfection. With this regimen the infection subsided and signs of activity had disappeared within 2-3 weeks. Follicles and corneal non-active opacities could be detected for weeks to some months after systemic treatment was finished. Twenty-three patients with chlamydial eye infection could be followed up until the condition healed, which took on average about two months after diagnosis. Nine patients had control cultures taken on average 13 days (range 2-30 days) after treatment, which were all negative.

STD patients. Of the 855 patients examined by genital and eye swabs for chlamydial infection $67 \%$ were males, of whom 104 had genital chlamydial infection. C. trachomatis was detected in genital swabs among 55 of 281 females. In no case was chlamydial eye infection demonstrated. The mean age of males with genital chlamydial infection was 27 years and 24 years for females with $C$. trachomatis infection.

Healthy adults. Swabs from the eyes for chlamydial isolation were obtained from 127 healthy employees. C. trachomatis was not isolated in any case. Of this group $80 \%$ were females. The mean age in the whole group was 20 years.

\section{Discussion}

Chlamydial eye infection was found with the highest frequency among young adults at 20 to 25 years of age. This is the same age at which genital chlamydial infection was most common among the VD patients. Chlamydial salpingitis and genital chlamydial infections in puerperal women in our series are also most prevalent at 15 to 25 years of age. ${ }^{1314}$ Although $C$. trachomatis was not uncommon at these ages among the ophthalmological patients, it was not isolated from the eyes of any of the $855 \mathrm{VD}$ patients, of whom 159 had genital chlamydial infection. In patients with chlamydial eye infection more than half of those examined had concomitant genital chlamydial infection. In the VD patients we detected genital chlamydial infection in $18 \%$ of male patients and $20 \%$ of females. In earlier studies we found genital infection by $C$. trachomatis in $25 \%$ of the female patients at the VD clinic, in $5 \%$ of women at a health care centre, ${ }^{15}$ and in $2.4 \%$ of puerperal women. ${ }^{14}$

These figures demonstrate a much higher frequency of genital chlamydial infection among the ophthalmological patients with chlamydial eye infection than for the average population and underline the venereal nature of chlamydial eye disease. Asymptomatic carriers of $C$. trachomatis in the eyes seem to be uncommon in VD patients and healthy adults and would suggest that, when present, chlamydial eye infection is generally accompanied by disquieting symptoms from the eyes. Therefore these patients are often seen by the ophthalmologist and not by the venereologist or the gynaecologist, though genital symptoms may also be present. It is also noteworthy that other pathogenic bacteria were not disclosed in 26 of the 28 cases with chlamydial conjunctivitis. In two cases bacterial cultures were not performed.

One case with chlamydial conjunctivitis was encountered in which non-venereal transmission may have occurred. Chlamydial eye infection developed in a nursing mother a few days after chlamydial conjunctivitis had been confirmed in her 2-weeks-old baby. Autoinfection in the mother is possible, but eye-to-eye transmission between infant and mother also seems likely.

It seems that chlamydial eye infection is manifested in a minority of patients with chlamydial infection. In our laboratory, which has about 700 to 800 chlamydial isolates a year, 7 to 10 come from adults with chlamydial conjunctivitis. A rough estimate would then be that between 1 and $2 \%$ of chlamydial infections are accompanied by eye symptoms.

Symptoms were unilateral in the majority of cases, indicating that autoinfection from one eye to the other is limited. In two cases unilateral pseudoptosis without other signs of cranial nerve paralysis was the most conspicuous symptom. The pseudoptosis was due to subtarsal swelling and follicles and disappeared completely after treatment.

It was also evident that symptoms could persist for weeks and months if a correct diagnosis and treatment were delayed. In our material the proportion of patients with longstanding eye symptoms may be exaggerated, as these patients were more likely to be examined for chlamydial infection. On the other hand they show that chlamydial conjunctivitis may have a lingering course, involving repeated consultations, if the true nature of the condition is not 
suspected and proper therapy therefore not given. After effective treatment corneal inactive opacities persisted, in some cases for several weeks before they resolved in all the 23 patients whom it was possible to follow up.

Treatment must aim at eradication of chlamydial infection in both the eyes and the genital tract. This goal can be achieved by systemic therapy with either tetracyclines or erythromycin, probably with similar efficacy. In pregnant women or nursing mothers tetracyclines must be avoided. Erythromycin is therefore the obvious choice in these special situations. Consorts to patients with chlamydial conjunctivitis are very likely to have genital chlamydial infection. Chlamydial salpingitis and perhaps infertility in the female is probably the most ominous aspect of chlamydial infection, which should be remembered when dealing with infections by $C$. trachomatis.

\section{References}

1 Jones BR. Ocular syndromes of TRIC virus infection and their genital significance. BrJ Vener Dis 1964; 40: 3-18.

2 Viswalingam ND, Wishart MS, Woodland RM. Adult chlamydial ophthalmia (paratrachoma). Br Med Bull 1983; 39: 123-7.

3 Darougar S, Jones BR. Trachoma. Br Med Bull 1983; 39: 117-22.
4 Grayston JT, Wang SP. New knowledge of chlamydiae and the diseases they cause. J Infect Dis 1975; 132: 87-105.

5 Jones BR, Collier LM. Inoculation of man with inclusion blenhorrhae virus. Ann NY Acad Sci 1962; 98: 212-26.

6 Jones B, Al-Hyssain K, Dunlop E. Infection of the eye and genital tract by TRIC agent: III Ocular syndromes associated with infection of the genital tract by TRIC agent. Rev Int Trach Pathol Ocul Trop Subtrop 1965; 42: 27-43.

7 Dawson CR, Schachter J. TRIC agent infections of the eye and genital tract. Am J Ophthalmol 1967; 63 (suppl): 1288-98.

8 Schachter J, Rose L, Meyer KF. The venereal nature of inclusion conjunctivitis. Am J Epidemiol 1967; 85: 445-52.

9 Rees E, Tait IA, Hobson D, Byng RE, Johnson FWA. Neonatal conjunctivitis caused by Neisseria gonorrhoeae and Chlamydia trachomatis. Br J Vener Dis 1977; 53: 173-9.

10 Julianelle LA, Harrison RW, Lange A. Studies on inclusion blennorrhea. Am J Ophthalmol 1938; 21: 1230-41.

11 Thygeson $\mathrm{P}$, Stone W. Epidemiology of inclusion conjunctivitis. Arch Ophthalmol 1942; 27: 91-122.

12 Huntemüller, Paderstein. Chlamydozoen-Befunde bei Schwimmbad-conjunctivitis. Dtsch Med Wochenschr 1913; 36: 63-66.

13 Osser S, Persson K. Epidemiologic and serodiagnostic aspects of chlamydial salpingitis. Obstet Gynecol 1982: 59: 206-9.

14 Persson K, Rönnerstam R, Svanberg L, Holmberg L. Maternal and infantile infection with chlamydia in a Swedish population. Acta Paediatr Scand 1981; 70: 101-5.

15 Persson K, Persson K, Hansson $\mathrm{H}$, et al. Prevalence of nine different micro-organisms in the female genital tract. A comparison between women from a venereal disease clinic and from a health control department. Br J Vener Dis 1979; 55: 42933. 